DSC measurement of Auln sample

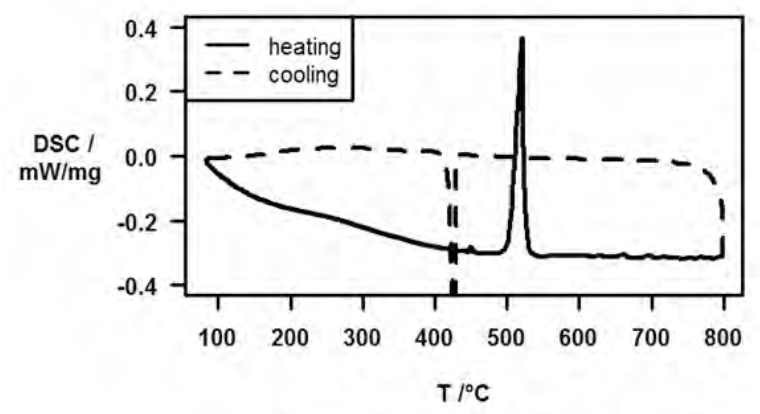

DSC measurement of PdBi sample

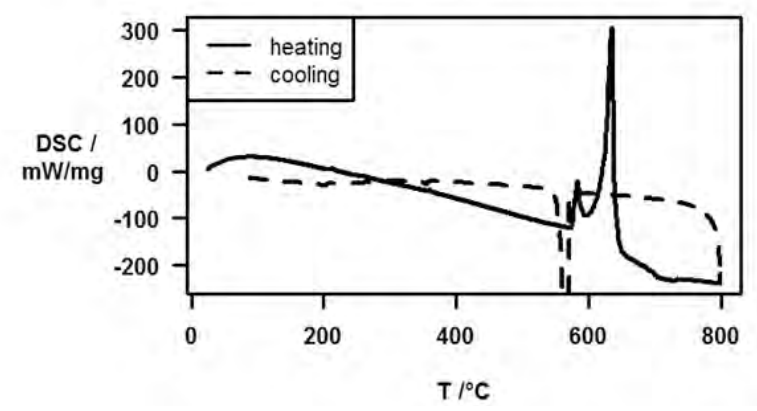

References:

[1] Folkers, L. C. \& Simonov, A. \& Wang, F. \& Lidin, S. (2018), Inorg. Chem., 57, 2791 - 2796.

[2] Schubert, K. \& Rösler, U. \& Kluge, M. \& Anderko, K. \& Härle,

L. (1953), Naturwissenschaften, 40, 34.

[3] Zhuravlev, N. N. (1957), Zhurnal Eksperimental'noi i

Teoreticheskoi Fiziki', 5, 1064 - 1072.

Keywords: Aperiodics, Intermetallics, Synchrotron Data
MS27-05

\section{Adventures in modulation: derivatives of barluenga's reagent}

Amber L. Thompson ${ }^{1}$, Lewis C. F. Morgan ${ }^{1}$, Yejin Kim¹, Emma J. McKinley ${ }^{1}$, Jack N. Blandy ${ }^{2}$, Claire A. Murray ${ }^{3}$, Kirsten E.

Christensen

1. Chemical Crystallography, Department of Chemistry, University of Oxford., Oxford, United Kingdom

2. Department of Chemistry, University of Oxford., Oxford, United Kingdom

3. Diamond Light Source Ltd, Harwell Science and Innovation Campus., Didcot, Oxfordshire., United Kingdom

email: amber.thompson@chem.ox.ac.uk

Single-crystal X-ray diffraction is a powerful technique: as the technique has developed data collections have been getting faster and faster, and structure determination is becoming more routine. There are an increasing number of non-expert users collecting data, solving and refining structures before publishing their own results. However, with the advent of higher intensity laboratory X-ray sources, easier access to synchrotron radiation and more sensitive detectors, more and more molecular structures are showing alien features beyond the realms of conventional crystallography [1].

Though the concept of modulated structures is now fairly well established in solid state chemistry, the abundance of reported modulated molecular materials is less than might be expected. There are a number of probable reasons for this, including the fact that the synthetic chemist is primarily interested in connectivity and proof of what they have made, so they are not curious about additional peaks seen in a diffraction pattern. When tweaking the synthesis and/or changing crystallisation conditions makes the curious extra features disappear, this is typically the chosen route and the original structure is casually swept under the carpet.

When a concerted effort is made to study the appearance of additional satellite reflection and relating it to changes within the structure, valuable extra information can be gained concerning molecular packing and the crystalline state. One such example is found in Barluenga's reagent, $\mathrm{IPy}_{2} \mathrm{BF}_{4}(\mathrm{Py}$ $=$ pyridine) [2], which has been shown to exhibit a transient modulated phase on cooling (see image). Systematic studies on derivatives of Barluenga's reagent have been carried out in which the pyridine is replaced with 2,4,6-trimethyl pyridine (collidine, Coll), iodine with bromine and the $\mathrm{BF}_{4}$ anion is replaced with other small anions including $\mathrm{ClO}_{4}$ and $\mathrm{PF}_{6}[3]$. Through these changes to the chemistry we can begin to understand how the crystal structure and the diffraction pattern are related and why satellite peaks may appear, with the aim of improving our understanding of the mechanism governing the appearance of modulation in molecular materials. 

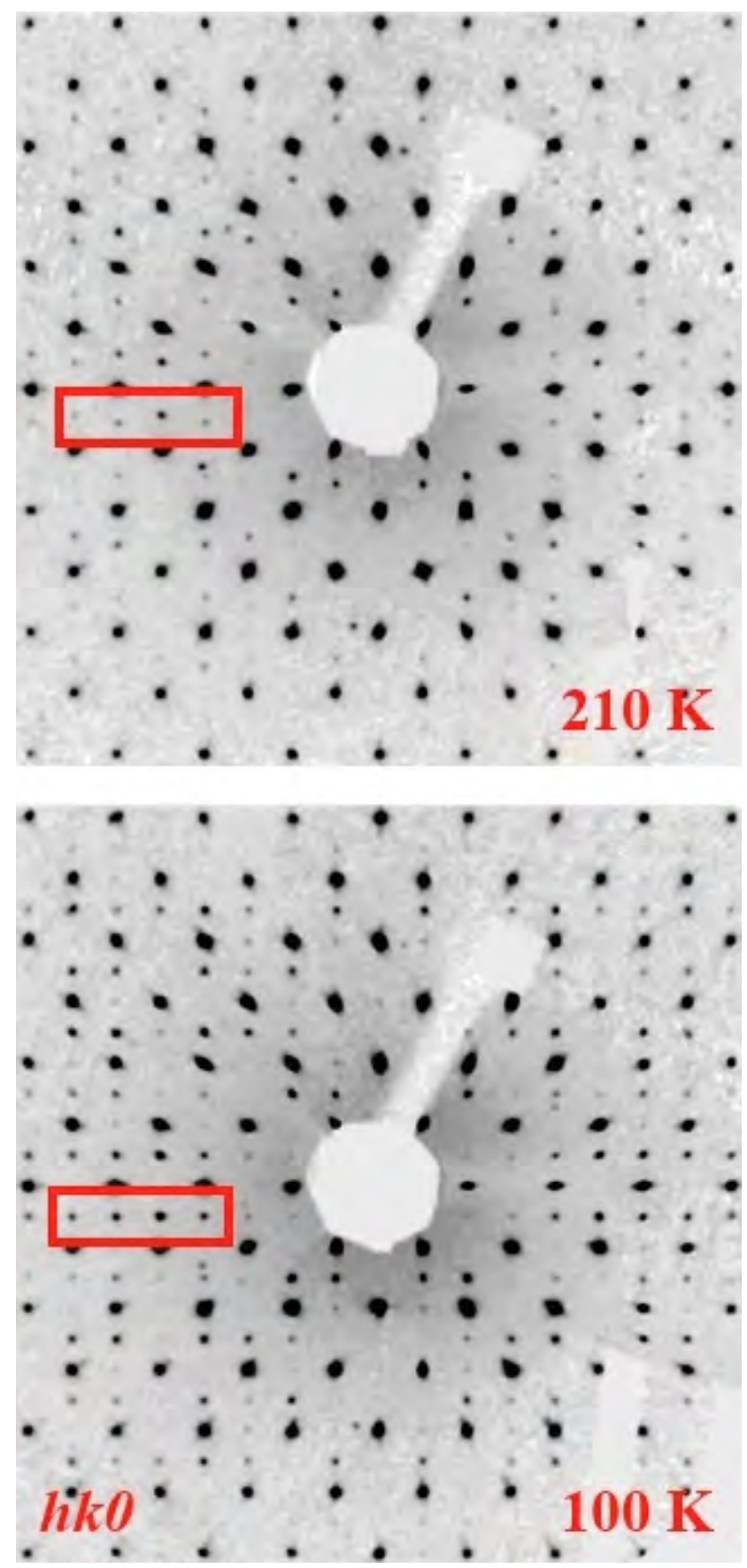

References:

[1] Bond, A. D. (2012). CrystEngComm. 14, 2363-2366.

[2] Chalker, J. M. , Thompson, A. L. \& Davis, B. G. (2010). Organic Syntheses, 87, 288-298.

[3] Kim, Y., Mckinley, E. J., Christensen, K. E., Rees, N H., \& Thompson, A. L. (2014). Cryst. Growth Des., 14, 6294-6301.

Keywords: Modulation, Barluenga's Reagent, Variable Temperature

\section{MS28 Magnetic order: methods and properties}

Chairs: Dr. Francoise Damay, Prof. José L. García-Muñoz

\section{MS28-01}

\section{On the symmetry of incommensurate magnetic structures}

J. Manuel Perez-Mato ${ }^{1}$, Luis Elcoro ${ }^{1}$, Gotzon Madariaga ${ }^{1}$, Mois I. Aroyo $^{1}$

1. Dept. Fisica de la Materia Condensada, Facultad de Ciencia y

Tecnologia, Universidad del Pais Vasco, UPV/EHU, Bilbao, Spain email:jm.perezmato@gmail.com

The concept of symmetry in materials science trascends the role of being just a mere mathematical or geometrical property. In general, the symmetry of a crystal, within a certain thermodynamical phase, is an intrinsic physical property of the phase (phase transitions can occur without a symmetry change, but a symmetry change cannot happen without a phase transition!). In this sense, the symmetry of a crystal can be considered a thermodynamical property, which will be preserved by any possible physical atomistic mechanisms that may be active in the system as long as no phase transition occurs.

The symmetry of aperiodic crystals (structures that are ordered, but lack periodicity), is given by the so called superspace symmetry groups. As required of any well-defined generalized symmetry, these symmetry groups satisfy the above mentioned physical condition of being an intrinsic property that is necessarily preserved within a thermodynamical phase.This generalized symmetry formalism was introduced in the seventies to describe the symmetry properties of incommensurately modulated crystals, and in the next decade was further extended and applied to quasicrystals. Nowadays the formalism is fully established, and since decades superspace groups are used in a routinary and systematic way to characterize incommensurate and quasicrystalline structures.

Although the superspace formalism is easily extensible to incommensurate magnetic structures, its application to magnetic systems has remained scarce until recently. Only in the last years the development of specially adapted computer tools, and in particular, the extension of the JANA refinement program to magnetic structures has changed the situation. Using these freely available tools, superspace symmetry formalism can now be applied and this allows an efficient and rigorous description of incommensurate magnetic structures making full use of their symmetry. An additional step in this direction has been the development in the Bilbao Crystallographic Server of a small database of such structures (www.cryst.ehu.es/magndata), where the magnetic superspace group of each structure have been identified and their efficient decription using this symmetry group is shown in detail.

In this talk I will review the concepts of magnetic superspace symmetry and its application. The relation with the traditional representation method will be discussed, stressing their complementarity. Some examples retrieved from 